\title{
A large 3D physical model: a tool to investigate the consequences of ground movements on the surface structures
}

\author{
M. Caudron ${ }^{1, \mathrm{a}}$, B.Hor ${ }^{1,2}$, F. Emeriault ${ }^{2}$ and M. Al Heib ${ }^{1}$ \\ ${ }^{1}$ INERIS, DRS, Parc technologique Alata, 60550 Verneuil en Halatte, France \\ ${ }^{2}$ Université de Lyon, INSA-Lyon, LGCIE, 69621 Villeurbanne, France
}

\begin{abstract}
Soil subsidence of various extend and amplitude can result from the failure of underground cavities, whether natural (for example caused by the dissolution of rocks by underground water flow) or man-made (such as mines). The impact of the ground movements on existing structures (houses, buildings, bridges, etc...) is generally dramatic. A large small-scale physical model is developed in order to improve our understanding of the behaviour of the building subjected to ground subsidence or the collapse of cavities. We focus on the soil-structure interaction and on the mitigation techniques allowing reducing the vulnerability of the buildings (structures).
\end{abstract}

\section{Introduction}

INERIS has been working for many years on the "ground movements" risk caused by old mines. Several research tasks are followed concerning the stability of the cavities, the prediction of the ground movements, the behaviour of the building subjected to this hazard, mitigation techniques...

A large small-scale physical model is developed in order to improve our understanding of the behaviour of the building due to ground subsidence or collapse. This paper focuses on the soilstructure interaction and on the mitigation techniques allowing reducing the vulnerability of structures.

This paper is composed in the first part of the presentation of the physical model, the measurement facilities and the basic hypothesis. The second part will detail the building model from the prototype considerations to the simplification done for the small-scale model. We developed then two similar tests, the first one presents the case of a ground subsidence in greenfield condition (no surface structure) while the second one presents the case with the building model: a loaded slab. Three positions of the structure with respect to the cavity position are considered in order to determine the effect of these positions. The effects are detailed for the behaviour of the soil and the building in terms of displacements, strains and loss of support.

\footnotetext{
a e-mail : matthieu.caudron@ineris.fr
} 


\section{Bibliography}

\subsection{Mining subsidence \& soil-structure interaction}

The subsidence is caused by the collapse of anthropic or natural underground cavities. This phenomenon can be very prejudicial for the structures and infrastructures on the surface and for the population. Empirical rules exist for the estimation of the building damages caused by such ground movements (...). But they are limited by the context of their definition. There is very few relationships to determine the intensity of the building damages caused by ground movements and taking into account the soil-structure interactions.

Several research projects are focused on the study of the ground-structure interactions phenomena under the effect of the soil movements ([1-7]).

While previous works [8] have allowed shedding some light on the interest of taking into account the soil-structure interaction. This paper will focus on the conception of a new small-scale physical model intended for the study of mining subsidence and the consequences on surface building. Previously, a 2D small-scale physical model was used [9]. However, the 2D nature of this model was a too important limit for the expected results preventing any reliable interpretation to the reality.

\subsection{Physical model for mining subsidence}

Very few physical models have been used to study the effects of ground movements caused by the mining process or by the collapse of old mine. We can mention block caving apparatus from [10, 11]. This model is dedicated to the study the block caving exploitation scheme and not to the ground movement rising to the surface. Few models are focusing on the phenomena corresponding to the formation of a subsidence trough. Genis [12] studied the effect of an earthquake on the stability of an old coalmine located in Japan. They showed that depending on the geometry of the cavity, the failure may take place in the pillars or at the mine ceiling. Dyne (1998) designed a model, trap door type, in order to represent the formation of sinkholes in an ancient coalmine in Pennsylvania. The model was a very simple one with a trap door of four different widths and a single layer of sand as overburden.

For these different works, the potential damages caused by the subsidence on the surrounding buildings were not taken into account.

Ren [13] achieved several tests in centrifuge concerning subsidence features of ground and wall rock due to underground mining. Few details are mentioned in the different papers published on the parameters and the hypothesis made to this model.

On this subject, several small-scale physical models have been realized in the field of tunnel. For example, Nakai [7] and Shahin [14] use a 2D physical model for studying the influence of the position and nature of the foundation on a building in response to tunnelling-induced settlements.

Model tests have been performed in the context of EU research project "QUAKER" [15] to study the influence of several buildings, considering different geometries, weight and foundation systems, on the path followed by a fault activated by the Kocaeli earthquake. Very interesting results are obtained, showing a more or less important bifurcation of the fault when a building is located close the path obtained in greenfield condition. The bifurcation can even become very important in the case of heavy building or when a deep foundation system is used.

\section{The Apparatus}

\subsection{Hypothesis and conception}

Physical model must respect similitude laws; the first part of the hypothesis comes from the laws of similitude. It is very difficult, if not impossible to achieve a perfect similitude with a small-scale model. Since our physical model is designed to be used in a $1 \mathrm{~g}$ environment (earth gravity) and not 
in a centrifuge, it will be difficult for quantitative interpretations of the experimental data to be made.

The geometry scale factor may range up to $1 / 50$. With a size of $2 \mathrm{~m}$ by $3 \mathrm{~m}$ in the horizontal plan for a height up to $1 \mathrm{~m}$, we would be able to represent a soil block as large as $100 \mathrm{~m}$ by $150 \mathrm{~m}$ with a height of 50m. A key limitation due to the $1 \mathrm{~g}$ environment is that for high scale factor values, the difference between the achieved values of stresses and the normally scaled ones become very large and might cause some important differences.

Considering underground mines as cavities at the origin of the ground movements, caused by their collapse, the maximum depth of $50 \mathrm{~m}$ may appear like an important limit to this model. However, we chose not to model the cavity itself but only the equivalent void raising the ground surface. This is achieved by the use of an electric jack with a vertical moving trap-door. The physical model is designed to be equipped with 48 jacks at most, in a configuration of 6 rows of 8 columns.

The model is still in an early stage of development, only one jack is installed. The cross-section of the actuator is limited to $250 \times 250 \mathrm{~mm}^{2}$, corresponding up to $12.5 \times 12.5 \mathrm{~m}^{2}$ in the prototype scale. The apparatus is indeed limited to localized phenomena: sinkhole or collapse/subsidence of smallextent. When more jacks would be installed, various shapes of collapse/subsidence would be able to be modeled..

\subsection{The soil}

The sand of Fontainebleau, well-known by the actors of physical modeling in geotechnics, is used. Category NE 34 is chosen whose granulometry and characteristics are presented in figure 1 and table 1 .

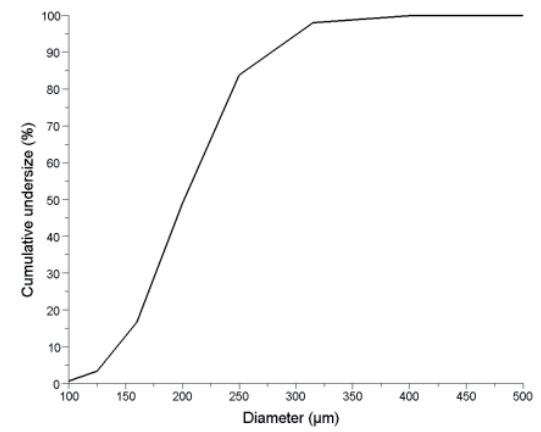

Table 1. Mechanical characteristics of the sand

\begin{tabular}{|c|c|c|c|c|}
\hline & Density & E (MPa) & $\varphi\left(^{\circ}\right)$ & $\mathrm{c}(\mathrm{kPa})$ \\
\hline Sand & 1.6 & $5-20$ & $32-34$ & $0-2$ \\
\hline
\end{tabular}

Fig. 1. Granulometry of the Fontainebleau sand

\subsection{Monitoring}

Measurement is achieved by the mean of stereo digital imagery. This allows monitoring the whole top surface of the ground and more especially where ground movements happen. A commercial product is chosen, Vic3D from Limess $\mathrm{Gmbh}$, being very effective in the task of computing the movements with the grains of sand showing very low contrast on the photos.

Two cameras, 4MP each, are used. They allow a maximal frequency of capture of 8 images/second at full resolution, with the possibility to reach 30 images/s with a $1 \mathrm{MP}$ resolution. They have to be calibrated before starting a test by the use of a test pattern. A good calibration allow obtaining very precise measures with an error of $1 / 100$ of a pixel in good conditions: that's $10 \mu \mathrm{m}$ when 1 pixel is equal to $1 \mathrm{~mm}$. In the tests presented later in this paper, we have a ratio close to 2 pixels per millimeter. But, due to the nature of the sand, constituted of small particles and so not a continuous media, the corresponding error is around 0.13 pixel giving $0.065 \mathrm{~mm}$ : this is quite a good performance [16].

One disadvantage of this method of monitoring is the huge volume of data created by a single test. With a volume of $8 \mathrm{Mo}$ per capture (two images of $4 \mathrm{Mo}$ each) and considering the full speed 
frequency of capture, it represents near 2 Go of raw data to be stored each minute. For a full test and with the exploitation files for the digital correlation process, this corresponds to a total between 30 and $40 \mathrm{Go}$.

\subsection{The building model}

A building model was created to investigate the effects of the soil-structure interactions during the formation of a subsidence trough and the effects of this ground movement on the structure.

The geometry chosen for the building was inspired from the database existing on the building damaged by mine subsidence in the east of France, for instance the Lorraine basin [17]. A house is considered, whose characteristics are shown in figure 2 . While it is not possible to make directly a 3D realistic small-scale model of this structure, we have to simplify it. For the first test series, we chose to consider only an elastic behavior and to use an equivalent slab. The followed process to simplify the building and to obtain the small-scale model is presented on figure 2 .

Three steps were made for the simplification procedure: first, the equivalent slab is determined such as the bending stiffness and the axial stiffness of this slab are quite equivalent to the 3D structure. Then we choose to reduce the stiffness, in both directions, in order to exacerbate the strains in the structure. Both stiffnesses are approximately halved. From this very simple structure at prototype scale, a small scale model is conceived by applying the scale factors.

As shown in figure 3, two parts are used for the structure model. The first one is a hollow slab while the second one is composed of lead powder in a plastic bag. This allows the model to present a stiffness and a stress transmitted to the ground equivalent to the prototype's ones.

This small scale model is laying on the ground without any foundation system which limits the interaction between the soil and the structure. However, this procedure makes it very simple to reproduce on several tests. The next small-scale building model should integrate a foundation system so as to respect a configuration closer to the reality.

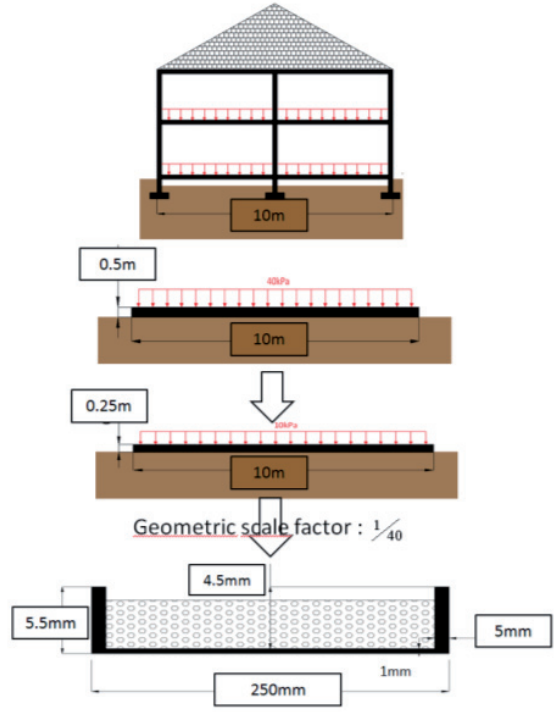

Fig. 2. Simplification procedure of the $3 \mathrm{D}$ building to an equivalent small-scale slab.

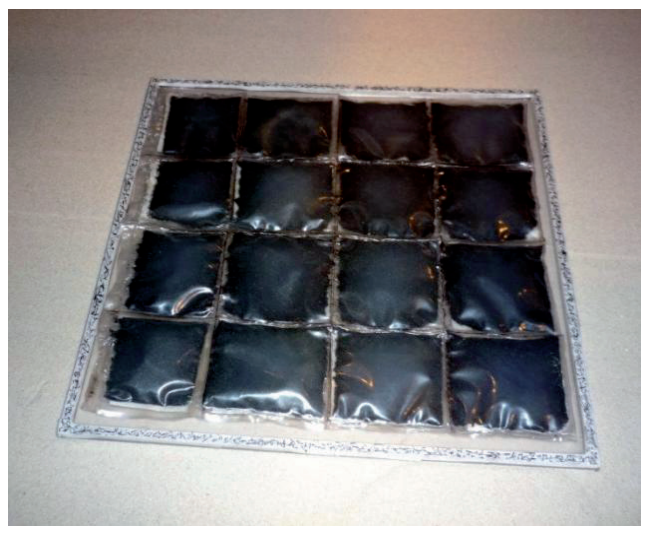

Fig. 3. The resulting small-scale structure model composed of a hollow slab and small bags of lead powder representing the load.

\subsection{Achievement of a test}

The sand is manually placed in the apparatus by layers of $15 \mathrm{~cm}$ thick. Each layer is compacted by a compacting tool equipped by $15 \mathrm{~cm}$ long needles in order to obtain a certain level of density, 
repeatable for each different test. This is repeated until the total height of the soil layer is reached, in our case, $30 \mathrm{~cm}$. Afterwards, a $130 \mathrm{~cm}$ wide metallic rule is used to obtain the right level on the whole ground surface.

A snapshot is then taken by both cameras to insure that the ground surface is flat enough with a difference less than $5 \mathrm{~mm}$ in altitude between the highest and the lowest points. The building model is then placed carefully on the ground surface, its position depends on the modeled case (see part 4 of this paper).

The displacement acquisition by the two cameras is then started with a frequency of $0.5 \mathrm{~Hz}$. The motion program of the jack is launched at the same time a snapshot is taken by the cameras. The jack is moved down at a speed of $0.125 \mathrm{~mm} / \mathrm{s}$ for a total of $30 \mathrm{~mm}$.

At the end of the test, the displacements of the ground and the structure are computed by the DIC (Digital Image Correlation) program.

\section{Results of the test series}

The first test campaign is dedicated to study the influence of the building position relatively to a subsidence trough. After the presentation of the reference result, the subsidence trough is in greenfield condition, the results from the parametric study will be presented.

\subsection{Greenfield condition}

Four identical tests were performed in order to ensure a good level of repeatability of the tests. As shown on figure 4, the greenfield trough is symmetric. The average global characteristics are summarized in table 2. Differences from one test to another are not very important, but cannot be neglected.

Table 2. Geometric characteristics of the surface ground displacement

$\left(\mathrm{S}_{\max }\right.$ : maximal subsidence, $\mathrm{i}$ : distance to point of inflexion, $\mathrm{R}_{\min }$ : minimal radius of curvature, $\alpha$ : maximal slope, $\varepsilon_{\mathrm{h}}$ : maximal horizontal strain)

\begin{tabular}{|c|c|}
\hline Characteristics & Average \\
\hline Smax & $24 \mathrm{~mm}$ \\
\hline $\mathrm{i}$ & $100 \mathrm{~mm}$ \\
\hline Rmin & $1.7 \mathrm{~m}$ \\
\hline slope $\alpha$ & $22 \%$ \\
\hline$\varepsilon \mathrm{h}$ & $5 \%$ \\
\hline
\end{tabular}

\subsection{Parametric study}

Three positions (A, B, C) are used for the building model. They are defined with respect to the main component of the subsidence trough. Position A corresponds to the building being on the extension area and position B puts the building completely in the compression area, position $\mathrm{C}$ is where the slope is the greatest. Figure 5 presents these three different positions. Three particular axes are then defined for the exploitation of the results which pass through the center of the building to the center of the subsidence trough.

Four tests are performed for each position of the building, as for the greenfield condition. Results are then presented for two points of view. First the displacements measured at ground surface will be compared in order to identify the effect of the building on the ground behavior. Secondly the strains in the building model will be analyzed. 




Fig. 4. Displacements in Greenfield condition

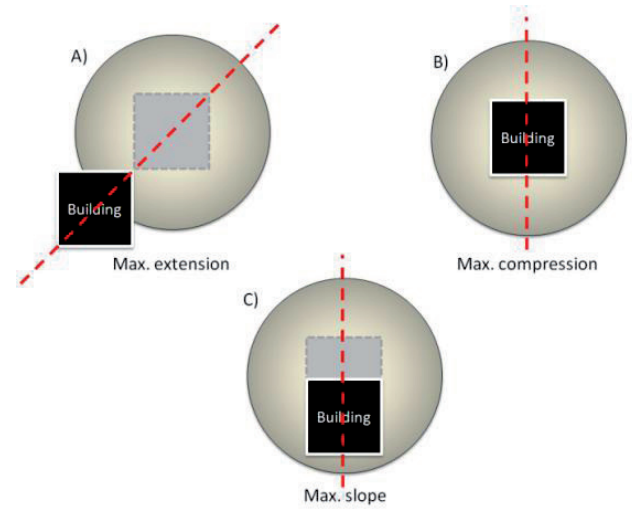

Fig. 5. Building position for the parametric study

\subsection{Ground behaviour}

The building produces important differences in the soil displacement at ground surface. The trough, formerly symmetric in greenfield condition, shows clearly some dissymmetry with the presence of the building. However, the building being in B position, centered in the subsidence trough, remains symmetric. Two examples of ground displacement curves are shown on figure 6, corresponding to the axis plotted for the positions A \& $\mathrm{C}$ of the building.

It appears that the soil movements are reduced due to the soil-structure interaction. Different areas may be distinguished, depending on the relative displacement of the ground to the building model. In the center part of the trough, the ground falls off the building due to a more important displacement, while on the other side of the building, its rotation causes another detachment.


Fig. 6. Behaviour of the ground for two different positions of the building (left: pos. C; right: pos. A).

For the $\mathrm{C}$ position, three different behaviors are identified. For the part laying close to the center of the trough, a loss of support appears, thus the soil behavior is not very disturbed by the soilstructure interaction. For the center part of the structure, the contact between ground and building is kept and so important modifications are visible in the behavior: the slope of the soil is largely reduced and the horizontal displacements are restrained to the average horizontal displacement of the building. For the last part, another loss of support appears where the rigid body rotation of the building engenders uplift.

For the A position, the chosen axis for the behavior of ground and the building reveals a very important loss of support concerning two third of the appearing length. In fact, this is not so important due to the fact that the structure is supported on its sides, not visible here. The impact of the soil-structure interaction relative to the vertical displacement is less important than for position 
C. For the horizontal one, the shape of the displacement curve is very close to the one in greenfield condition. The values of maximal displacements are the same.

\subsection{Consequences on the building}

The optical setting allows measuring the movements and the strains of the building. Those measures are synthesized in table 3. It can be seen that the measured strains are very small comparing to the strain of the soil. Several reasons are identified:

- The elastic behavior chosen for the building model;

- The fact that the building is just laying on the ground surface;

- The stiffness of the building is relatively too important.

The soil-structure interaction is thus clearly identifiable: the strains measured on the building are very different from those measured in greenfield condition. The behavior of the soil is notably modified due to the presence of the building. The influence of the soil-structure interaction depends moreover on the position of the building relatively to the subsidence trough. In a general manner, the strains measured on the structure model are smaller than those in greenfield condition. The main differences are shown for the horizontal strain and for the bending radius.

Table 3. Comparison between the strains determined in greenfield condition and using the building model.

\begin{tabular}{|c|c|c|c|}
\hline Case & $\begin{array}{c}\text { Horiz. strain } \\
(\%)\end{array}$ & $\begin{array}{c}\text { Bending } \\
\text { radius (m) }\end{array}$ & Slope (\%) \\
\hline Greenfield (pos. C) & 5 & -1.06 & 9.5 \\
\hline SSI (pos. C) & 0.08 & -25 & 6.4 \\
\hline Greenfield (pos. A) & 10 & -1.67 & 10.4 \\
\hline SSI (pos. A) & 0.04 & -9.42 & 7.1 \\
\hline
\end{tabular}

\section{Conclusions and prospects}

The study presents a new 3 D physical model allowing the simulation of ground movements caused by the collapse of underground cavities. A simple structure model was designed in order to represent an individual house, was used for two purposes: to observe the evolution of the ground movements subjected to the presence of the building, and the behavior of the building itself, and, to shed some light on the importance of the soil-structure interaction.

As a conclusion for this study, we would like to emphasize the fact that these tests represent the first step of a more global research for the consequences of ground movements on building, by taking into account the soil-structure interactions. It was established than the soil-structure interaction phenomenon must not be neglected and that it depends greatly on the relative position of the building to the subsidence trough.

Still, many technical improvements should be brought to the small-scale physical model. The initial state of the sandy soil must be clarified and several jacks would be set up to increase the variety of subsidence trough geometry that the apparatus is able to achieve.

However, in parallel, the structure model should be improved in order to represent more closely a real building. The main points are the design of the foundation system and the upper part of the structure. A new test series should be then conducted with the final objectives to study the performance of mitigation techniques. 


\section{Acknowledgment}

The authors would like to thank the French Ministry for Environment, Sustainable Development and the Sea for the partial founding of this research program.

\section{Bibliography}

[1] Standing, J. Impact of underground works on existing infrastructure. Post-Mining'08, Nancy (France) (2008). Invited Lecture.

[2] Lee, Y. \& Bassett, R. Influence zones for 2D pile-soil-tunnelling interaction based on model test and numerical analysis. Tunnelling and Underground Space Technology (2007) 22:325342.

[3] Sung, E.; Shahin, H.; Nakai, T.; Hinokio, M. \& Makoto, Y. Ground behavior due to tunnel excavation with existing foundation. Soils and Foundations, (2006), 2:189-207.

[4] Abbass-Fayad, A. Modélisation numérique et analytique de la montée de cloche des carrières à faible profondeur. Etude de l'interaction sol-structure due aux mouvements du terrain induits par des fontis. Institut National Polytechnique de Lorraine, (2004).

[5] Deck O., Al Heib M., and Homand F. Taking the soil-structure interaction into account in assessing the loading of a structure in a mining area. Engineering Structure (2003) 25:435-448.

[6] Burd H.J., Houlby G., Augarde C., Liu G. Modelling tunneling-induced settlement of masonry building. Proc. Instn. Civ. Engrs Geotech. Engng, (2000), pp 17-29.

[7] Nakai, T.; Xu, L. \& Yamazaki, H. 3D and 2D model tests and numerical analyses of settlements and earth pressures due to tunnel excavation. Soils and Foundations, (1997), 37:3142.

[8] Caudron M., Emeriault F. \& Al Heib M. Contribution of the experimental and numerical modeling to the understanding of the soil-structure interaction during the event of a sinkhole. ECSMGE (2007), Madrid.

[9] Caudron M., Emeriault F., Kastner R. \& Al Heib M. Sinkhole and soil-structure interactions: Development of an experimental model. Int. Conf. on Physical Modeling in Geotechnics, Hong-Kong, (2006), pp 1261-1267.

[10] Castro R., Trueman R. \& \&Halim A. A study of isolated draw zones in block caving mines by means of a large 3D physical model. IJRM (2007), 44:860-870.

[11] Trueman R., Castro R, \& Halim A. Study of multiple draw-zone interaction in block caving mines y means of a large 3D physical model. Int. J. of Rock Mechanics and Mining Sciences (2008) 45:1044-1051.

[12] Genis, M. \& Aydan, O. Assessment of dynamic response and stability of an abandoned room and pillar underground lignite mine. $12^{\text {th }}$ IACMAG, October (2008). pp 3899-3906.

[13] Ren W., Wang Y., Bai S. \& Ge X. Research on deformation and subsidence characters of ground and wall rock due to underground mining by model testing. $6^{\text {th }} I C P M G$. 2006. pp 1527-1533.

[14] Shahin, H.; Nakai, T.; Hinokio, M.; Kurimoto, T. \& Sada, T. Influence of surface loads and construction sequence on ground response due to tunnelling. Soils and Foundations, (2004), 44:71-84.

[15] Bransby M., Davies M. \& El Nahas A. Centrifuge modeling of normal fault foundation interaction. Bull. Earthquake Eng. (2008), 6:585-605.

[16] White D., Take W. and Bolton M. Soil de formation measurement using particle image velocimetry (PIV) and photogrammetry. Geotechnique, (2003), 53:619-631.

[17] Saeidi A., Deck O. \& Verdel T. Development of a simulator of damage for evaluation of the vulnerability of buildings in subsidence zones. Post-Mining (2008), France, pp 1-12. 